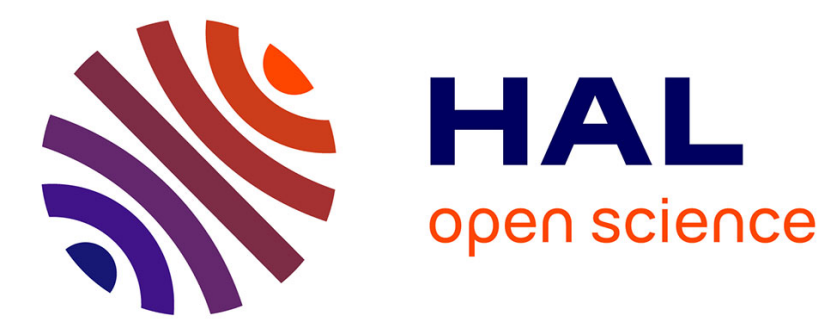

\title{
Effect of the number of patches in a multi-patch SIRS model with fast migration on the basic reproduction rate
}

Etienne Kouokam, Pierre Auger, Hassan Hbid, Maurice Tchuente

\section{To cite this version:}

Etienne Kouokam, Pierre Auger, Hassan Hbid, Maurice Tchuente. Effect of the number of patches in a multi-patch SIRS model with fast migration on the basic reproduction rate. Acta Biotheoretica, 2008, 56 (1-2), pp.75-86. 10.1007/s10441-008-9036-y • ird-00296726

\section{HAL Id: ird-00296726 \\ https://hal.ird.fr/ird-00296726}

Submitted on 15 Jul 2008

HAL is a multi-disciplinary open access archive for the deposit and dissemination of scientific research documents, whether they are published or not. The documents may come from teaching and research institutions in France or abroad, or from public or private research centers.
L'archive ouverte pluridisciplinaire $\mathbf{H A L}$, est destinée au dépôt et à la diffusion de documents scientifiques de niveau recherche, publiés ou non, émanant des établissements d'enseignement et de recherche français ou étrangers, des laboratoires publics ou privés. 


\title{
Effect of the number of patches in a multi- patch SIRS model with fast migration on the basic reproduction rate
}

\author{
Etienne Kouokam ${ }^{1}$, Pierre Auger ${ }^{2,4}$, Hassan Hbid $^{3}$ and Maurice Tchuente ${ }^{1,2,4}$ \\ ${ }^{1}$ Laboratory MAT-IRD, Department of Computer Science, Faculty of Science, University of \\ Yaounde I, P.O. Box 812 Yaounde, Cameroon \\ ${ }^{2}$ IRD - UR GEODES, 32 avenue Henri Varagnat, 93143 Bondy Cedex, France \\ ${ }^{3}$ Department of Mathematics, University of Menara, Morocco \\ ${ }^{4}$ Institut des Systèmes Complexes (IXXI), ENS-Lyon, 46 allée d’Italie 69364 Lyon Cedex 07, \\ France \\ kouokam@yahoo.com, Pierre.auger@bondy.ird.fr, Maurice.tchuente@ens-lyon.fr
}

\begin{abstract}
We consider a two-patch epidemiological system where individuals can move from one patch to another, and local interactions between the individuals within a patch are governed by the classical SIRS model. When the time-scale associated with migration is much smaller than the time-scale associated with infection, aggregation methods can be used to simplify the initial complete model formulated as a system of ordinary differential equations. Analysis of the aggregated model then shows that the two-patch basic reproduction rate is smaller than the 1 patch one. We extend this result to a linear chain of $\mathrm{P}$ patches $(\mathrm{P}>2)$. These results are illustrated by some examples for which numerical integration of the system of ordinary differential equations is performed. Simulations of an individual based model implemented with a multi-agent system are also carried out.
\end{abstract}

Keywords: SIRS model, two-patch system, linear chain of patches, fast migration, aggregation of variables, basic reproduction rate, individual based model, multiagent simulations.

\section{Introduction}

A simple approach for the analysis of the spread of infectious diseases, is the classical SIR model initially studied by Hamer (1906), Ross (1911), Kermack and McKEndrick (1927). The main assumption of this model is that a population in which a pathogenic agent is active, can be divided into three distinct compartments corresponding to different epidemiological status. The first compartment is made of the susceptible (S), i.e. the healthy individuals who can catch the disease and are therefore said to be susceptible to infection. The second compartment is composed of infective (I) individuals who are infected and are capable of transmitting the infection. The third compartment consists of recovered (R) individuals who, after being infected, have recovered from the infection. It is important to note that in this model, recovery implies permanent or temporary immunity. In the latter case the removed individuals return to the susceptible compartment and we obtain the SIRS model illustrated by the transfer diagram of figure $1 \mathrm{~b}$. In the case where recovery implies permanent immunity, we obtain the SIR model illustrated in figure $1 \mathrm{a}$. In both cases, it is generally assumed that the total population is constant. As a consequence, at any time $t$, the number of susceptible $\mathrm{S}(\mathrm{t})$, infective $\mathrm{I}(\mathrm{t})$ and recovered $\mathrm{R}(\mathrm{t})$ are such that $\mathrm{S}(\mathrm{t})+\mathrm{I}(\mathrm{t})+\mathrm{R}(\mathrm{t})=$ $\mathrm{N}$. Hereafter, we shall concentrate on the SIRS model. 
An infection can occur through a contact between a susceptible and an infective individual. It is assumed that the various classes of individuals are uniformly mixed, i.e. every pair of susceptible and infective individuals has equal probability to come into contact. The system of ordinary differential equations below describes the time evolution of the population density variables:

$$
\left\{\begin{array}{l}
\frac{d S}{d t}=-\beta I S+\gamma R \\
\frac{d I}{d t}=-\delta I+\beta I S \\
\frac{d R}{d t}=\delta I-\gamma R
\end{array}\right.
$$

where $\beta>0$ is the proportion of contacts inducing infection, called the infection rate, $\delta$ is the recovery rate and $\gamma$ is the rate at which recovered individuals become susceptible again.

For any $\mathrm{t}$, the total population $\mathrm{N}=\mathrm{S}(\mathrm{t})+\mathrm{I}(\mathrm{t})+\mathrm{R}(\mathrm{t})$ remains constant. Substituting $\mathrm{N}-\mathrm{S}(\mathrm{t})$ - I(t) to $\mathrm{R}(\mathrm{t})$ leads to the following reduced system.

$$
\left\{\begin{array}{l}
\frac{d S}{d t}=-\beta I S+\gamma(N-S-I) \\
\frac{d I}{d t}=-\delta I+\beta I S
\end{array}\right.
$$

It is well known that this couple of ordinary differential equations has two equilibriums: the disease-free equilibrium $(N, 0)$ and the endemic equilibrium $\left(S^{*}, I^{*}\right)=\left(\frac{\delta}{\beta}, \frac{\gamma\left(N-S^{*}\right)}{\gamma+\beta S^{*}}\right)$.

A critical threshold for the study of the dynamics of SIRS models is the basic reproduction rate which is the average number of secondary infections that can arise from a single primary case. For the analytical model introduced above, $R_{0}=\frac{\beta N}{\delta}$. Moreover, local stability analysis shows that :

if $\mathrm{R}_{0}<1$, then the disease-free equilibrium is locally asymptotically stable, i.e. starting from an initial primary infection in a host population composed of sane individuals, the infection dies out, or equivalently no epidemic occurs.

$>$ if $\mathrm{R}_{0}>1$, then the endemic equilibrium is locally asymptotically stable, i.e. an initial primary infection leads to an epidemic which spreads and persists.

In this paper, we consider an environment consisting of two patches with migration between them. On each patch, local epidemiological dynamics is described by the previous SIRS model with the same parameters. Dispersion among patches is represented by migration with constant proportions of migrants. We shall show that if the migration process is much faster than the epidemiological process, i.e. if the time-scales associated with migration and epidemics are not of the same order, then aggregation methods can be used to derive an equivalent simplified model describing the dynamics of the total sane and infective populations. Moreover we show that $\mathrm{R}_{0}^{2} \leq \mathrm{R}_{0}$, where $\mathrm{R}_{0}^{2}$ is the basic reproduction rate of the aggregated model corresponding to the system of two patches connected by fast migration, and $\mathrm{R}_{0}$ is the previous basic reproduction 
rate which corresponds to the case of a single patch or to a system of two patches when the migration are not allowed and the total population is initially present on the same patch. To be precise, we shall refer to:

- $\mathrm{R}_{0}$ as the single or 1-patch basic reproduction rate,

- $\mathrm{R}_{0}^{2}$ as the 2-patch basic reproduction rate.

Furthermore, we exhibit situations where $\mathrm{R}_{0}>1$, i.e. an initial primary infection can invade and persist in the host population on a single isolated patch, and $\mathrm{R}_{0}^{2}<$ 1, i.e. the disease cannot persist when the population extend on two patches with fast migration. This result is extended to the case of linearly connected patches. Illustrations are given through examples for which numerical integration of the systems of ordinary differential equations is performed. We also introduce an individual based model implemented with a multi-agent system, which leads to simulations that are quite consistent with the numerical results derived from the analytical model.

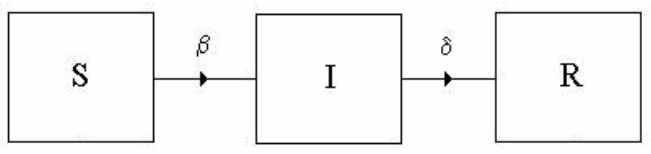

(a)

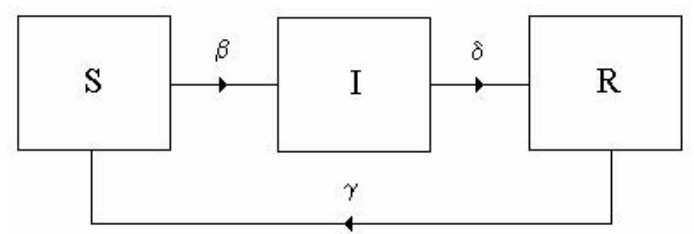

(b)

Figure 1: Transition graph for the SIR (a) and SIRS (b) models

\section{Description of the Model}

\subsection{The complete model}

Now, we consider a two-patch system with the same parameters. We assume that individuals can move from one patch to the other.

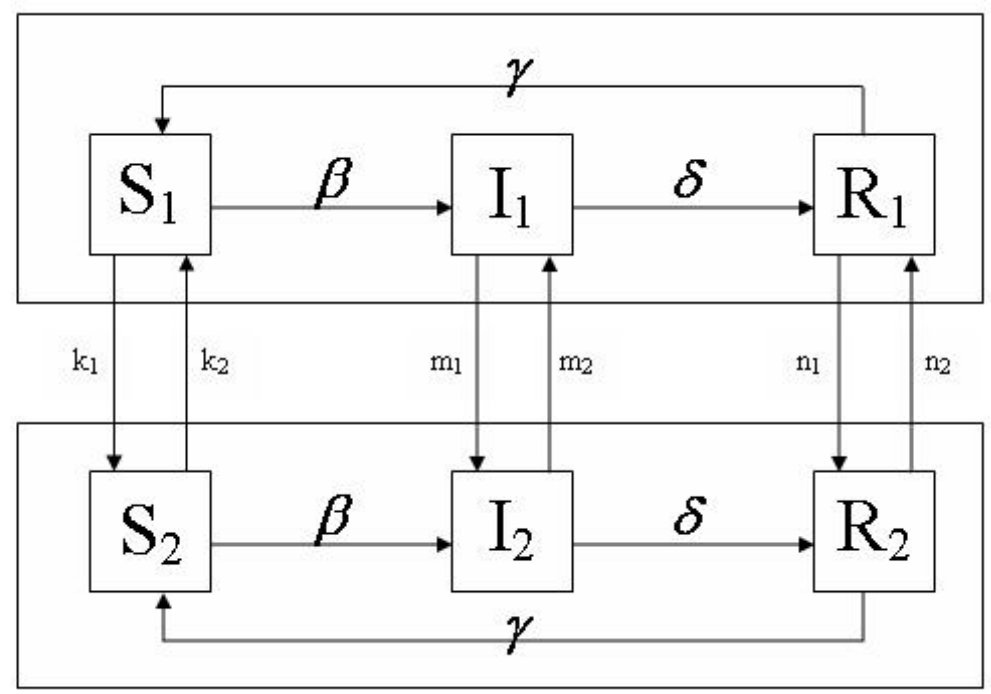

Figure 2: Transition graph in a two-patch system for the SIRS model

We consider the two-patch SIRS model illustrated in figure 2. Each patch corresponds to the classical SIRS model with the same parameters $(\beta, \delta, \gamma)$. This 
is consistent with the fact that in real situations, the characteristics of the disease do not depend on the patch. The vertical arrows represent the migration process which is assumed to proceed with constant proportions. Let us denote $\left(\mathrm{S}_{1}, \mathrm{~S}_{2}\right)$, $\left(I_{1}, I_{2}\right)$ and $\left(R_{1}, R_{2}\right)$ respectively the populations of susceptible, infective and recovered individuals, with index indicating the patch number. Assuming that the time scales for migration and infection are $\tau$ and t respectively, with $t=\varepsilon \tau$, the combined effect of the two processes involved in the two-patch model can be described as follows:

$$
\left\{\begin{array}{l}
\frac{d S_{1}}{d \tau}=\left(k_{2} S_{2}-k_{1} S_{1}\right)+\varepsilon\left(-\beta I_{1} S_{1}+\gamma R_{1}\right) \\
\frac{d I_{1}}{d \tau}=\left(m_{2} I_{2}-m_{1} I_{1}\right)+\varepsilon\left(\beta I_{1} S_{1}-\delta I_{1}\right) \\
\frac{d R_{1}}{d \tau}=\left(n_{2} R_{2}-n_{1} R_{1}\right)+\varepsilon\left(\delta I_{1}-\gamma R_{1}\right) \\
\frac{d S_{2}}{d \tau}=\left(k_{1} S_{1}-k_{2} S_{2}\right)+\varepsilon\left(-\beta I_{2} S_{2}+\gamma R_{2}\right) \\
\frac{d I_{2}}{d \tau}=\left(m_{1} I_{1}-m_{2} I_{2}\right)+\varepsilon\left(\beta I_{2} S_{2}-\delta I_{2}\right) \\
\frac{d R_{2}}{d \tau}=\left(n_{1} R_{1}-n_{2} R_{2}\right)+\varepsilon\left(\delta I_{2}-\gamma R_{2}\right)
\end{array}\right.
$$

In these equations, the first terms represent the migration of individuals between the two patches and the second terms represent the infection process, where $\varepsilon$ is a dimensionless parameter. Hereafter, we assume that the migration time-scale is much faster than the infection time-scale, i-e, $\varepsilon<<1$.

\subsection{The aggregated model}

When the migration process is much faster than the infection process, we can use aggregation methods to get a reduced model. This aggregated model describes the evolution over time of the total susceptible, infective and recovered populations and is obtained in two steps. First, we neglect the infection process since $\varepsilon<<1$ and we study the migration process. This is equivalent to setting $\varepsilon=0$ in (3). The fast equilibrium is quite easy to compute and is given by the following expressions:

$$
\begin{array}{lll}
S_{1}^{*}=\frac{k_{2}}{k_{1}+k_{2}} S=v_{1}^{*} S & I_{1}^{*}=\frac{m_{2}}{m_{1}+m_{2}} I=\mu_{1}^{*} I & R_{1}^{*}=\frac{n_{2}}{n_{1}+n_{2}} R=\eta_{1}^{*} R \\
S_{2}^{*}=\frac{k_{1}}{k_{1}+k_{2}} S=v_{2}^{*} S & I_{2}^{*}=\frac{m_{1}}{m_{1}+m_{2}} I=\mu_{2}^{*} I & R_{2}^{*}=\frac{n_{1}}{n_{1}+n_{2}} R=\eta_{2}^{*} R
\end{array}
$$

In these expressions, $v_{1}^{*}$ (resp. $v_{2}^{*}$ ) is the proportion of susceptible individuals at the fast equilibrium on patch 1 (resp. 2). Similarly, $\mu_{1}^{*}$ (resp. $\mu_{2}^{*}$ ) and $\eta_{1}^{*}$ (resp. $\eta_{2}^{*}$ ) are respectively the proportions of infective and recovered individuals on patch 1 (resp. 2).

It is easily seen that

$$
v_{1}^{*}+v_{2}^{*}=1, \mu_{1}^{*}+\mu_{2}^{*}=1 \text { and } \eta_{1}^{*}+\eta_{2}^{*}=1
$$


Substituting this fast equilibrium into the complete model (3) leads to the following aggregated model governing the total susceptible and infective densities:

$$
\left\{\begin{array}{l}
\frac{d S}{d t}=-\phi I S+\gamma(N-S-I) \\
\frac{d I}{d t}=-\delta I+\phi I S
\end{array}\right.
$$

This aggregated model corresponds to a SIRS system where $S(t)=S_{1}(t)+S_{2}(t)$, $I(t)=I_{1}(t)+I_{2}(t), \mathrm{N}$ is the total density of the population and

$$
\phi=\beta\left(v_{1}^{*} \mu_{1}^{*}+v_{2}^{*} \mu_{2}^{*}\right)=\frac{\beta\left(k_{1} m_{1}+k_{2} m_{2}\right)}{\left(k_{1}+k_{2}\right)\left(m_{1}+m_{2}\right)}
$$

From (5), $v_{1}^{*}+v_{2}^{*}=1$. As a consequence, $v_{1}^{*} \mu_{1}^{*}+v_{2}^{*} \mu_{2}^{*} \leq \max \left(\mu_{1}^{*}, \mu_{2}^{*}\right) \leq 1$ Hence:

$$
\phi \leq \beta
$$

\subsection{Analysis of the aggregated model}

Following the classical results established for SIRS models, the aggregated model (6) has two equilibriums: the disease-free equilibrium $(N, 0)$ and the endemic equilibrium $\left(S^{*}, I^{*}\right)=\left(\frac{\delta}{\phi}, \frac{\gamma\left(N-S^{*}\right)}{\gamma+\phi S^{*}}\right)$

The basic reproduction rate of the aggregated model is $\mathrm{R}_{0}^{2}=\frac{\phi N}{\delta}$. As a consequence, as a result of Eq. (8), we always have:

$$
\mathrm{R}_{0}^{2} \leq R_{0}
$$

This shows that in case of fast migration, the 2-patch basic reproduction rate is always smaller or equal to the single-patch one. This is an interesting result which says that if the migration time-scale is much faster than the infection time-scale, then the dynamics of two connected patches with the same parameters is not identical to the dynamics of the single patch system. An interesting question concerns the existence of some particular parameters leading to an aggregated model which exhibits dynamics qualitatively different from the behaviour of the single patch system. This question will be answered in the next paragraph.

\subsection{Influence of the spatial distributions of susceptible and infective individuals on $\mathrm{R}_{0}^{2}$}

In this paragraph, we assume that we have a two-patch system with fast migration. The 2-patch basic reproduction rate $\mathrm{R}_{0}^{2}$ can be expressed in terms of the 1-patch basic reproduction rate and the proportions of susceptible and infective individuals on each patch as follows: 


$$
\begin{aligned}
\mathrm{R}_{0}^{2} & =\frac{N \beta\left(v_{1}^{*} \mu_{1}^{*}+v_{2}^{*} \mu_{2}^{*}\right)}{\delta} \\
& =\frac{N \beta}{\delta}\left(1-v_{1}^{*}-\mu_{1}^{*}+2 v_{1}^{*} \mu_{1}^{*}\right) \\
& =R_{0}\left(1-v_{1}^{*}-\mu_{1}^{*}+2 v_{1}^{*} \mu_{1}^{*}\right)
\end{aligned}
$$

Let us now consider a particular case where the 1-patch basic reproduction rate is bigger than 1 , i-e $\mathrm{R}_{0}>1$. In other words, we assume that if the total population is initialy located on a single patch and cannot migrate, the endemic equilibrium is stable. We shall now look for specific spatial distributions of susceptible and infective individuals at the fast equilibrium, that lead to a 2-patch reproduction rate which is lower than 1 . This will correspond to the behaviour of an aggregated system that is qualitatively different from that of the 1-patch model. This situation occurs when:

$$
\mathrm{R}_{0}^{2}=R_{0}\left(1-v_{1}^{*}-\mu_{1}^{*}+2 v_{1}^{*} \mu_{1}^{*}\right)<1
$$

For example, if we assume a fixed spatial distribution for infective individuals, in order to have a 2-patch reproduction rate smaller than 1, susceptible individuals should distribute among the two patches according to the following inequalities:

$$
\begin{aligned}
& \text { If } \mu_{1}^{*}<\frac{1}{2} \text {, then } v_{1}^{*}>\frac{\mu_{1}^{*}+\frac{\delta}{N \beta}-1}{2 \mu_{1}^{*}-1} \\
& \text { If } \mu_{1}^{*}>\frac{1}{2} \text {, then } v_{1}^{*}<\frac{\mu_{1}^{*}+\frac{\delta}{N \beta}-1}{2 \mu_{1}^{*}-1}
\end{aligned}
$$

This situation is illustrated in figure 3 where it is assumed that $\frac{\delta}{N \beta}<\frac{1}{2}$, i-e $\mathrm{R}_{0}>2$. One can distinguish regions where the 2-patch basic reproduction rate is either bigger or lower than 1 . These regions depend on the proportions of susceptible and infective individuals on patch 1.

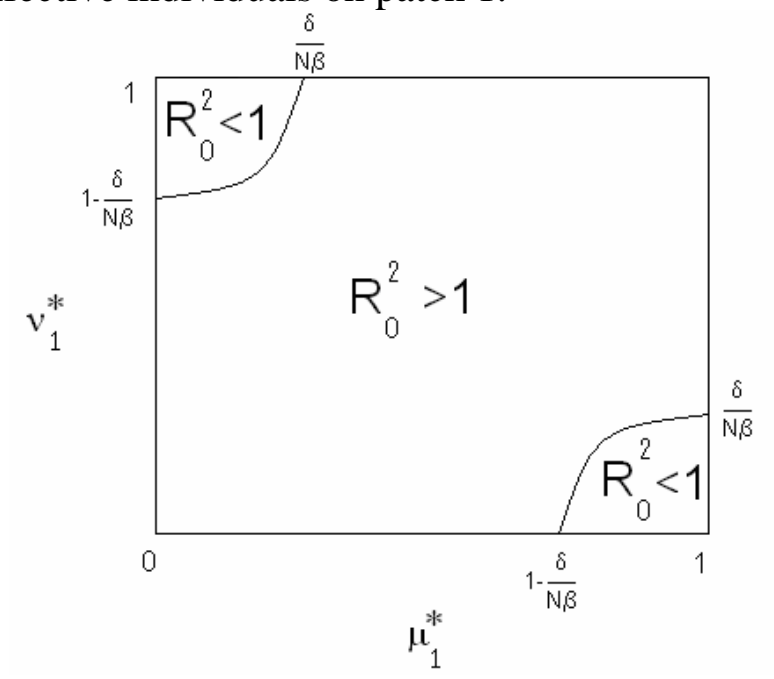

Figure 3: $\mathrm{R}_{0}>2$. Behaviour of $\mathrm{R}_{0}^{2}$ as a function of $v_{1}^{*}$ and $\mu_{1}^{*}$. 
There are two interesting regions, the upper left and the lower right, where the disease-free equilibrium is stable. These regions correspond to opposite spatial repartition for susceptible and infective individuals. In the upper left domain, most susceptible individuals are located on patch 1 and infective individuals on patch 2 . As a consequence, the possible contacts between the susceptible individuals and the infective are reduced considerably. The lower right region corresponds to the inverse situation. Thus, when infective and susceptible individuals are mostly located on different patches, the disease-free equilibrium is stable.

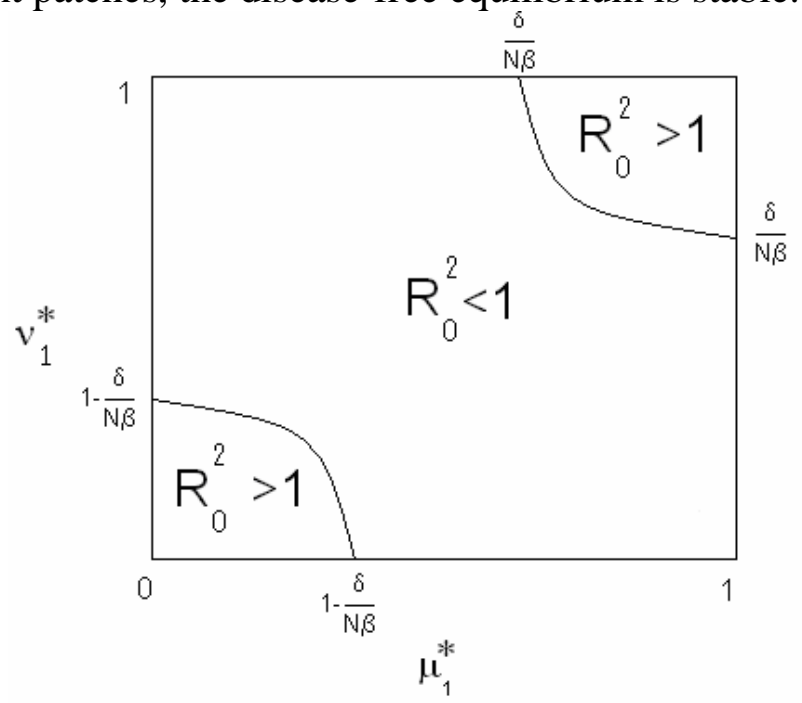

Figure 4: $1<\mathrm{R}_{0}<2$. Behaviour of $\mathrm{R}_{0}^{2}$ as a function of $v_{1}^{*}$ and $\mu_{1}^{*}$.

Figure 4 shows the behaviour of $\mathrm{R}_{0}^{2}$ as a function of $v_{1}^{*}$ and $\mu_{1}^{*}$ when $1<\mathrm{R}_{0}<2$. We obtain two regions, lower left and upper right, where the endemic equilibrium is stable in the two-patch system. This happens when both $v_{1}^{*}$ and $\mu_{1}^{*}$ are small or both $v_{1}^{*}$ and $\mu_{1}^{*}$ are close to 1 . In the first case, almost all individuals are located in patch 2 whereas in the second case, almost all individuals are located in patch 1 .

\subsection{The case of a system of linearly connected patches}

Let us consider a system of $\mathrm{P}$ linearly connected patches, $\mathrm{P}>2$, with fast migration between patches. The aggregation technique can be used to show that

$$
R_{0}^{P}=R_{0} \sum_{i=1}^{P} v_{i}^{*} \mu_{i}^{*} \text {, with } \quad \sum_{i=1}^{P} v_{i}^{*}=\sum_{i=1}^{P} \mu_{i}^{*}=1
$$

Where $R_{0}^{P}$ corresponds to the P-patch system. Hence, $R_{0}^{P} \leq R_{0}$. This shows that as for the 2-patch system, the global basic reproduction rate is lower than the 1-patch one. In the particular case where in the fast equilibrium, the infected as well as the susceptible individuals distribute equally among patches, i.e $v_{i}^{*}=\mu_{i}^{*}=\frac{1}{P}$ for $\mathrm{i}=1, \ldots, \mathrm{P}$, it can be shown that:

$$
R_{0}^{P}=\frac{R_{0}}{P}=\frac{\beta N}{\delta P}
$$

This shows that,

- If the total population is bigger, the epidemic is more likely to spread out. 
- If the number of patches is bigger, the stability of the disease-free state of the aggregated model is more likely to occur and the epidemic to vanish.

As we have seen for the two-patch system, the decrease of $R_{0}^{P}$ as the number of patches increases is even accentuated in the case of a fast equilibrium which yields a non balanced spatial distribution for susceptible and infective individuals.

This is a general trend in Ecology: the increase of the number of patches can have important consequences on the global behaviour of the system. This was shown for example in the context of a multi-patch host-parasitoid community. In the single patch case, when the Nicholson-Bailey model is used to describe local interactions between the two species, the host and parasitoid community cannot persist. However, in (Hassell et al. (1991a), (1991b)), the authors have shown that when the number of patches is increased, the community can persist. Our work shows similar results, i.e. that the number of patches has also an important effect on the dynamics of the system of connected patches. When the number of patches is increased, the dynamics is more likely to reach a disease free equilibrium.

\section{The Individual Based Model}

As explained in a recent paper (Gregory (2006)), the individual based-model is a philosophy that embraces the uniqueness of the individuals in a system. In this model, simulations are based on multiple individuals, each having its own set of variable parameters. In this way, it is possible to represent characteristics such as species, age, infectious status as well as events like movement and contacts between individuals. With this definition, SIRS systems appear to be good candidates for individual based models. Clearly, in our context here, we don't need the full power of the individual based model because of the simplicity of the behaviour of the individuals. However, the system under development is intended to be used in more complex epidemiological situations. This is why we have decided to develop a simulator based on the well known multi-agent environment Cormas (Bousquet et al. (1998)).

\subsection{Description of the simulator}

An agent needs attributes representing its patch number and its infection status. At each step, the simulation proceeds in two phases: the infection process, followed by the migration process.

The infection process is simulated by applying to all the susceptible individuals, the following procedure: pick a random number $\mathrm{r}$ in the interval $[0,1]$. If $r \leq \beta I$, then the corresponding individual becomes infected, otherwise it remains susceptible. The transfer from the infective to the recovered and from the recovered to the susceptible is treated according to a procedure similar to the technique used below for migration.

For the migration phase, the rate $\mathrm{k}_{1}$ of migration from patch 1 to patch 2 is simulated by applying in parallel to all the individuals of patch 1 the following procedure: pick a random number $r$ in the interval $[0,1]$ and move the concerned individual from patch 1 to patch 2 if $r \leq k_{1}$, otherwise that individual remains in patch 1. 


\section{Numerical Results}

A two-patch system is characterized by the following parameters: $\beta, \delta, \gamma, \mathrm{k} 1$, $\mathrm{k} 2, \mathrm{~m} 1, \mathrm{~m} 2, \mathrm{n} 1, \mathrm{n} 2, \mathrm{~N}$.

Case 1. This case corresponds to table 1 below. It can be shown that $\mathrm{R}_{0}^{2}<1$ and $\mathrm{R}_{0}>1$.

\begin{tabular}{|c|c|c|c|c|c|c|c|c|c|c|c|}
\hline Parameter & $\beta$ & $\delta$ & $\gamma$ & k1 & k2 & m1 & m2 & n1 & n2 & N & $\varepsilon$ \\
\hline Value & 0.005 & 0.9 & 0.9 & 0.2 & 0.9 & 0.8 & 0.1 & 0.25 & 0.35 & 350 & 0.01 \\
\hline
\end{tabular}

Table 1 : The parameter values of simulations

Figure 5.a presents the phase trajectories in the susceptible-infective phase plane for the aggregated model. It can be seen that the disease-free equilibrium $(\mathrm{S}, \mathrm{I})=(350,0)$ is stable and this is consistent with the fact that $R_{0}^{2}<1$. Figures 5.b and 5.c give the results of IbM simulations starting from $(S, I, R)=(100,250,0)$ for the aggregated model and from $\left(S_{1}, I_{1}, R_{1}\right)=(75,100,0)$ and $\left(S_{2}, I_{2}, R_{2}\right)=(25,150$, 0 ) for the complete model respectively. It can be seen that these curves match with the analytical results.

Figure 5.d presents the phase trajectories in the susceptible-infective phase plane for the 1-patch model. It can be seen that the endemic equilibrium $\left(S^{*}, I^{*}\right)=(71,139)$ is stable and this is consistent with the fact that $R_{0}>1$. Figure 5.e gives the curves of the susceptible and infective for the IbM simulation starting from $(S, I, R)=(100,250,0)$ in the 1-patch model. The convergence is not as obvious as in figures $5 b$ and $5 c$. However, the long term evolution presents oscillations around the endemic equilibrium point $\left(S^{*}, I^{*}\right)=(71,139)$.

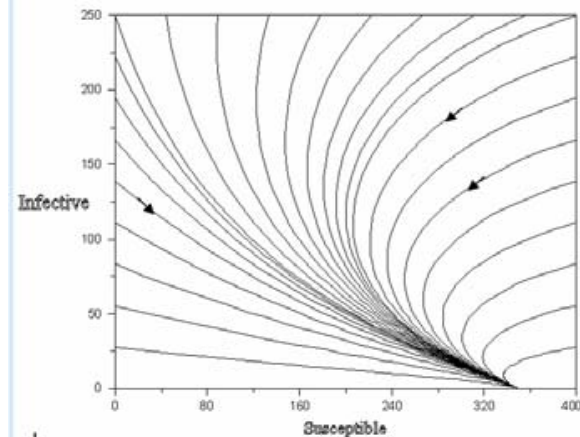

(a) Phase trajectories in the I-S plane for the aggregated model

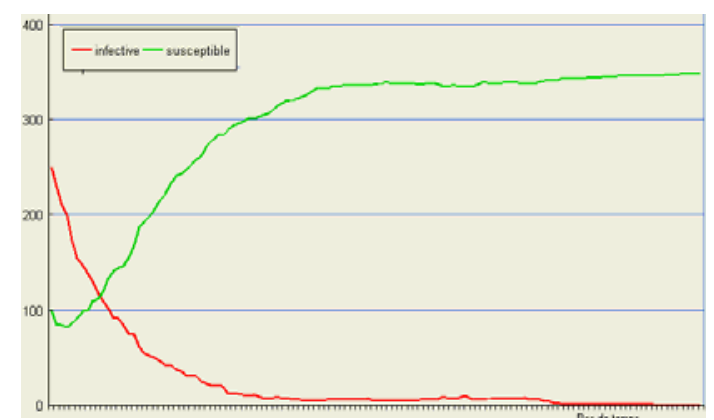

(c) An IbM evolution of infective and susceptible for the 2-patch complete model

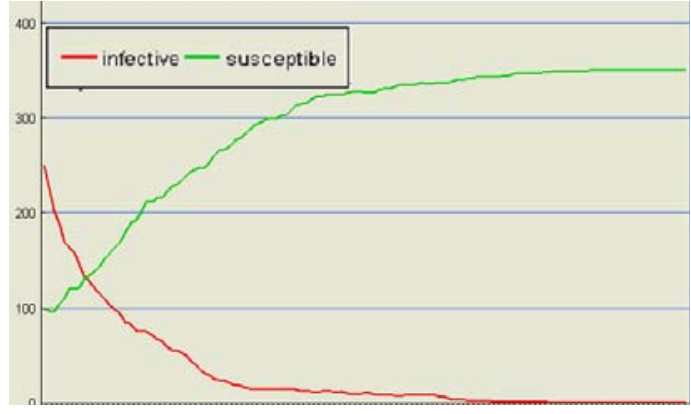

(b) An IbM evolution of infective and susceptible for the aggregated model

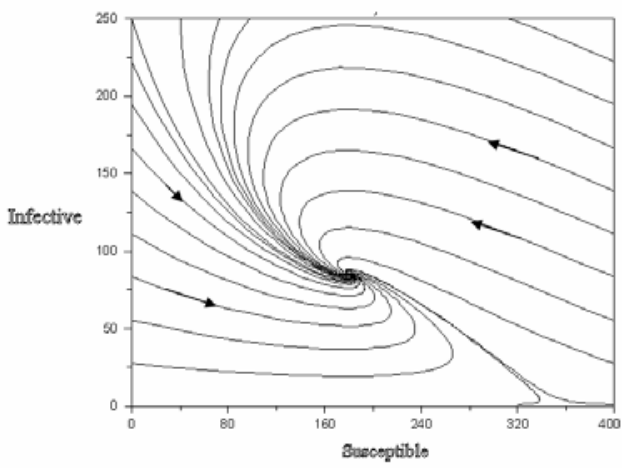

(d) Phase trajectories in the I-S plane for the local model 


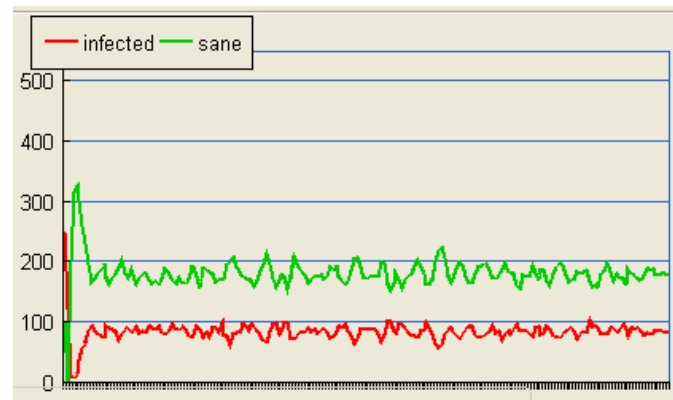

(e) An IbM evolution of infective and susceptible for the local model

Figure 5: Analytic and IbM simulations for case 1

Case 2 . This case is obtained from case 1 by changing $\beta$ to 0.05 . Here $R_{0}^{2}>1$ and $\mathrm{R}_{0}>1$.

Figure 6.a presents the phase trajectories in the susceptible-infective phase plane for the aggregated model. It can be seen that the endemic equilibrium is stable. Figure $6 \mathrm{~b}$ illustrates the validity of the IbM model. Indeed, the evolutions of figure $6 \mathrm{~b}$ show an example where the IbM simulation leads to the endemic equilibrium predicted by the aggregated model. Moreover, figures $6 \mathrm{c}$ and $6 \mathrm{~d}$ which give the evolution of the distributions of susceptible and infective in the two patches for the complete IbM model, are consistent with the fast equilibrium of the analytical aggregated model. Indeed, the sum of the susceptible of patch 1 (upper curve of figure 6c) and the susceptible of patch 2 (lower curve of figure 6c) corresponds to the susceptible in the total population (lower curve of figure 6b). Similarly, the sum of the infective of patch 1 (lower curve of figure 6d) and the infective of patch 2 (upper curve of figure $6 \mathrm{~d}$ ) corresponds to the infective in the total population (upper curve of figure $6 b$ ).

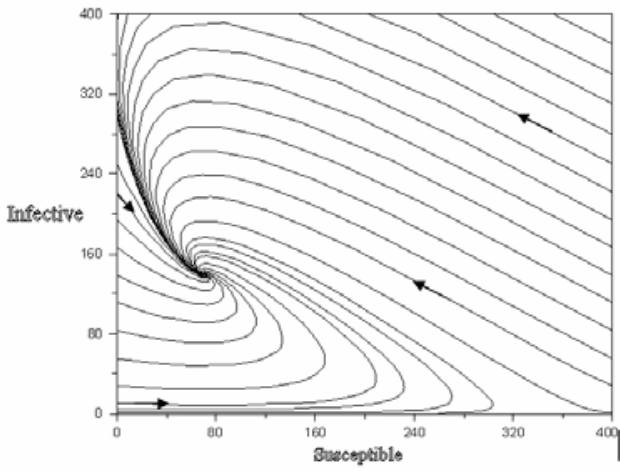

(a) Phase trajectories in the I-S plane for the aggregated model

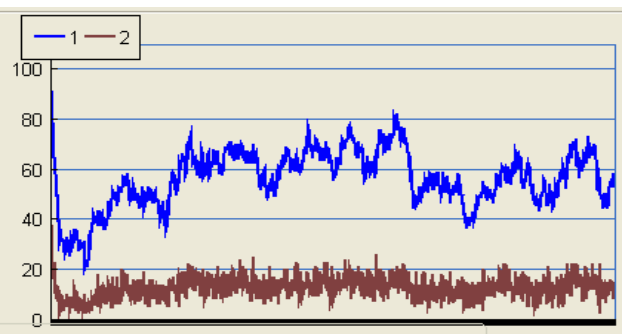

(c) IbM evolution of susceptible in patch 1 and 2 for the complete model

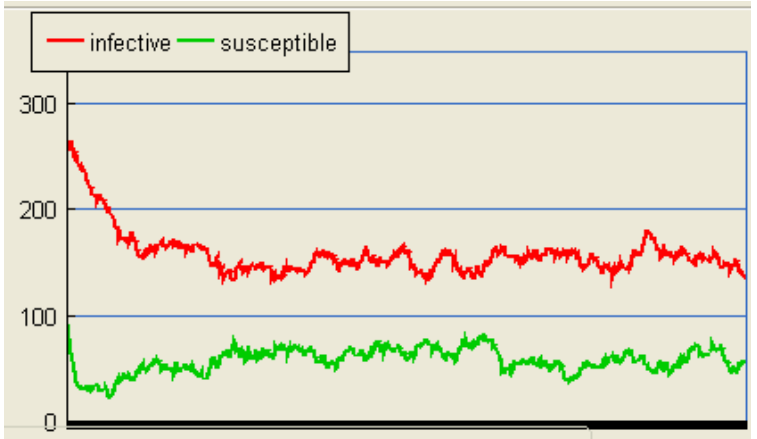

(b) IbM evolution of infective and susceptible for the aggregated model

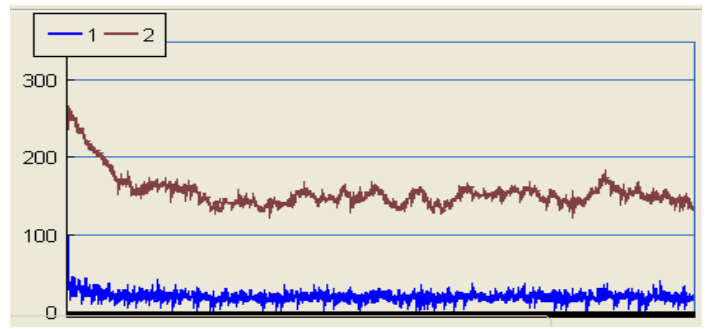

(d) IbM evolution of infective in patch 1 and 2 for the complete model 
Figure 6: Analytic and IbM simulations of aggregated and complete model for $R_{0}^{2}>1$

These simulations show clearly that for the examples treated, the IbM Model produces numerical results which are consistent with the analytical solutions. However, this consistency is more qualitative than quantitative. Indeed, as we have seen, analytical convergence can be represented in the IbM model by oscillations around the limit value.

\section{Conclusion}

In this paper, we have studied a system consisting of two SIRS patches with the same parameters. We have shown that if the time-scale associated with migration is much smaller than the time-scale associated with infection, aggregation methods can be used to simplify the initial complete model formulated as a system of ordinary differential equations. Analysis of the aggregated model then shows that the multi-patch basic reproduction rate is smaller than the 1-patch one. We have even exhibited examples where the endemic equilibrium is stable in the 1-patch model while the disease free equilibrium is stable in the multi-patch one. A structural change in the global behaviour of a system as one goes from a single to a multi-patch environment has also been observed in the Nicholson and Bailey host-parasitoid model by Hassell(1997, 2000a,b) and Lett (2003). In the future, we will consider a spatial SIRS model with patches distributed on a square lattice. The results of NguyenHuu (2006) indicate that in this context, we may see the emergence of new phenomena.

Another contribution of this paper is the design of an individual based model which leads to simulations that are qualitatively consistent with the analytical results. It is hoped that in circumstances where analytical results are not available, the simulations carried out with an IbM model can guide us in the study of the qualitative behaviour of patchy SIRS sytems.

\section{Acknowledgements}

We thank Gauthier Sallet for helpful remarks. Support from Institut de Recherche pour le Développement and Ecole Normale Supérieure de Lyon, laboratoire LIP and Institut des Systèmes Complexes is gratefully acknowledged.

\section{References}

Bousquet F., I. Bakam, H. Proton and C. Le Page, (1998), Cormas: Common-Pool Resources and Multi-agent Systems, in IEA/AIE '98: Proceedings of the $11^{\text {th }}$ International Conference on Industrial and Engineering Applications of Artificial Intelligence and Expert Systems: Tasks and Methods in Applied Artificial Intelligence, vol. 1416, Lecture Notes in Computer Science, 826-837, Springer.

Gregorya R., J.R. Saundersb, V.A. Saundersc (2006). The Paton individual-based model legacy. BioSystems 85, 46-54.

Hamer, W. H. (1906). Epidemic disease in England. The Lancet 1, 733-739.

Hassell, M. P. \& Wilson, H. B. (1997). The dynamics of spatially distributed host-parasitoid systems. In Spatial Ecology. The Role of Space in Population Dynamics and Interspecific Interactions, Tilman, D. \& Kareiva, P., eds, 75-110, Princeton University Press.

Hassell, M. P. (2000a). Host-parasitoid population dynamics. J. Anim. Ecol, 69, 543-566.

Hassell, M. P. (2000b). The Spatial and Temporal Dynamics of Host-parasitoid Interactions. Oxford University Press. 
Hassell, M. P., Comins, H. N. and R. M. May (1991a). Spatial structure and chaos in insect population dynamics. Nature 353, 255-258.

Hassell, M. P., May, R. M., Pacala, S. W. and Chesson, P. L. (1991b). The persistence of hostparasitoid associations in patchy environments, A general criterion. Am. Nat. 138, 568-583.

Kermack W. O., and McKendrick, A. G. (1927). Contributions to the mathematical theory of epidemics, part I. Proceedings of the Royal Society of London A115, 700-721.

Lett C., P. Auger and R. Bravo de la Parra (2003). Migration frequency and the persistence of host-parasitoid interactions. J. theor. Biol. 221, 639-654.

Nguyen-Huu T., C. Lett, J.C. Poggiale, P. Auger (2006). Effect of movement frequency on global host-parasitoid spatial dynamics with unstable local dynamics. Ecological Modelling 197, 290295.

Ross R., (1911). The Prevention of Malaria, 2nd edition. London. 